\title{
Editorials
}

\section{Trying to understand spinal anesthesia}

Quinn Hogan MD

A DMINISTERING safe and effective anesthesia requires not only observational vigilance, but also technology that enlightens us beyond that which we can perceive by natural senses. Starting in the 1970s, anesthetic practice entered a period with progressively more measuring and less guessing. We now take for granted that the cardiac rhythm will be readily evident by electrocardiography, intensity of neuromuscular block will be revealed by nerve stimulation, arterial oxygenation by pulse oximetry, and carbon dioxide elimination by capnometry. The quest for a monitor of anesthetic depth through electrophysiological indices is likewise driven by our communal discomfort at not having direct measures for crucial physiological functions during an anesthetic.

It is a similar dissatisfaction with opacity that repels some practitioners from regional anesthesia, since we have been operating in the dark regarding where nerves are, how the local anesthetic is distributed, and why there is so much variability in results. This accounts for the collective sigh of relief with the recent arrival of ultrasound imaging for peripheral neural blockade.

Unfortunately, we remain in the dark regarding critical aspects of neuraxial blockade. One source of frustration is that a given local anesthetic dose will produce a highly variable extent of anesthesia in different subjects. We recognize that certain categories of patients may on average have more extensive blockade, such as pregnant, aged, or obese subjects. Still, we have no means of anticipating how an individual subject will behave, and therefore have no opportunity to apply measures that control spread of blockade, such as regulating dose, specific gravity, and patient position.

A first light was shined on this mystery by Rosenberg and others when they followed a clinical hunch and confirmed that a patient who receives repeat spinal anesthetics closely duplicates the same spread of sensory anesthesia each time. ${ }^{1,2}$ Since the influences of age or body mass index do not account for this finding, some other patient characteristic must critically influence spinal anesthetic spread. An obviously relevant physical parameter is cerebrospinal fluid (CSF) volume. Just as we adjust doses of intravenous anesthetic agents to the patient's size in order to achieve the desired concentrations in the circulation and at the effector site, it would be appropriate to adjust the dose of intrathecal local anesthetic to suit the volume that initially dilutes it. Direct measurement of the vertebral CSF volume only became possible with the invention of axial imaging, and findings using magnetic resonance imaging $(\mathrm{MRI})$ indeed confirm that there is a notably wide span of volumes of lumbar CSF, for instance a range of $21 \mathrm{~mL}$ to $74 \mathrm{~mL}$ in 25 typical subjects. ${ }^{3}$ It is easy to believe that such extraordinary differences must regulate anesthetic dilution and therefore extent of blockade, ${ }^{4}$ and that a particular patient's CSF volume would be very valuable to know prior to spinal anesthesia. Although newer MRI techniques are more efficient,${ }^{5}$ this is not yet a feasible approach for planning clinical care.

This issue of the Journal reports a commendable attempt by Arzola et al. ${ }^{6}$ to make use of readily available ultrasound technology to determine an index of CSF volume. It is disappointing that the dural diameter they measured by ultrasound did not correlate with block extent, but there are considerations that made their study particularly challenging. Because the surgical indication was Cesarean delivery, they sought a high block, and it is not entirely surprising that the peak block height clustered tightly around T2 and T3, due to certain non-uniform features of the subarachnoid space. The amount of CSF at thoracic levels is

CAN J ANESTH 2007/54: 8/pp 607-612

From the Department of Anesthesiology, Medical College of Wisconsin, Milwaukee, Wisconsin, USA.

Address correspondence to: Dr. Quinn Hogan, Department of Anesthesiology, Medical College of Wisconsin, 8701 Watertown Plank Road, Milwaukee, Wisconsin 53226, USA. E-mail: qhogan@mcw.edu 
diminished compared to lumbar and cervical levels, ${ }^{3}$ and the thoracic nerve roots are very slight compared to segments above and below. ${ }^{7}$ Thus, there is less anesthetic dilution per segmental unit of distance from the site of injection, and the roots are easily blocked due to their small size, both factors predicting efficient blockade of these segments. Rostral to this, however, the increased CSF volume results in greater dilution and the larger roots are harder to block, so this is a common boundary for maximal spread of blockade. In a study such as this with near-maximal blocks, an influence of variable CSF diluent volume may not play a noticeable role. A second limitation is the current level of image resolution available by ultrasound. Without being able to distinguish the dura from ligamentum flavum, nor the substantial epidural fat between them, ${ }^{8}$ important differences in diameter might have been obscured. Finally, the dural sac is not a simple circle in cross section, ${ }^{8}$ so volumes might differ while the anterior/posterior dural diameter stays constant.

While I look forward eagerly to the continuing evolution of imaging methods that will ultimately provide a CSF volume measure, it is important to consider what we will do once we get that information. First, will we know what local anesthetic concentration to target? Some relevant numbers have already been nailed down. In sensory neuron cell bodies that are removed from the dorsal root ganglion by enzymatic dissociation, the plasma membrane voltage-gated $\mathrm{Na}^{+}$ channels are blocked by lidocaine with $\mathrm{EC}_{50}$ concentrations of $100 \mu \mathrm{M}$ (equal to $23 \mu \mathrm{g} \cdot \mathrm{mL}^{-1}$ ). ${ }^{9}$ However this is not entirely relevant, since these isolated neurons lack the encircling layer of satellite cells that are present in the natural state within the ganglion. Furthermore, sensory neuron axons express a superabundance of channels, such that action potentials may be conducted even while most of the $\mathrm{Na}^{+}$channels are blocked. For these reasons, the lidocaine concentration needed to interrupt conduction of action potentials in excised dorsal roots has an $\mathrm{EC}_{50}$ of about $230 \mu \mathrm{M}\left(54 \mu \mathrm{g} \cdot \mathrm{mL}^{-1}\right)$, and full blockade requires over $500 \mu \mathrm{M}\left(115 \mu \mathrm{g} \cdot \mathrm{mL}^{-1}\right) \cdot{ }^{10}$ Since these studies use rat tissue rather than human, and since in vitro chambers are very different from the intrathecal environment, cellular research must ultimately be validated by investigations in humans. Here, the experimental data is regrettably scant and remarkably old, but nonetheless appears to be very accurate. Landmark work starting in the 1930s ${ }^{11-13}$ employed periodic sampling of CSF by catheter or malleable needle to map out anesthetic distribution and action, initially examining procaine, the popular agent of that day. Subsequent investigation of lidocaine spinal anesthetics ${ }^{14}$ found concentrations at the return of sensation $\left(1.07 \mathrm{mM}, 250 \mu \mathrm{g} \cdot \mathrm{mL}^{-1}\right)$ and movement $\left(1.40 \mathrm{mM}, 327 \mu \mathrm{g} \cdot \mathrm{mL}^{-1}\right)$ that agree very well with the concentrations for full blockade found in modern animal experiments. ${ }^{10,15}$ (All together, studies by early regional anesthesia investigators inspire admiration for how much they got right).

Even with this knowledge in hand, there is a second challenge. Could we create the desired neuronal anesthetic concentration if we knew the patient's CSF volume? The problem here is that CSF pharmacokinetics are remarkably complex ${ }^{16}$ due to the simultaneous action of multiple processes. Although drug distribution is facilitated by the pulsatile oscillations of the $\operatorname{CSF}^{17}$ (recognized as an important factor by Koster et al. ${ }^{13}$ in 1936), the CSF is not well-stirred. Rather, there is a steep fall-off in concentration with increasing distances from the point of injection. ${ }^{12,18,19}$ This feature contrasts with the pharmacokinetics of systemic drug administration, in which concentrations promptly become uniform throughout the blood volume. The influence of baricity further adds to nonuniform drug distribution in the CSF. The disposition of drugs injected into the CSF is also complex in the time domain, since transport of drug from the CSF into nervous tissue competes with vascular uptake and departure of drug into the epidural space. Finally, steady state conditions are not achieved in this dynamic system, and there are indications that the peak concentration that initially drives local anesthetic into the nerve roots may have greater influence on extent and duration of blockade than the instantaneous concentrations thereafter. ${ }^{14}$ Although there have been exercises characterizing CSF pharmacokinetic performance using multicompartmental models ${ }^{20,21}$ these ultimately rely on the post hoc empirical fit of an uncertain number of exponentials to the data.

One consistent finding after drugs are injected into the subarachnoid space is the tremendous interindividual variability in measured concentrations. In small groups of subjects, three- to tenfold differences in concentration are found after injection of a common dose, ${ }^{14,18,19,21,22}$ even during the first ten to $30 \mathrm{~min}$ during which initial mixing is achieved. ${ }^{23}$ I suspect that this is in part due to the highly variable CSF volume, but also to the inhomogeneity of the space. Rather than the open chamber shown in common depictions and glass models, the posterior subarachnoid space is subdivided by plentiful membranes ${ }^{24-27}$ that are best appreciated in nonembalmed autopsy specimens. Anesthetic injected into this complex, multichambered system might enter the general pool of lumbar CSF, or in other cases may remain relatively 
sequestered, resulting in different degrees of dilution.

These observations predict that it will be difficult to calculate a dose of local anesthetic that will produce exactly the desired distribution and duration of spinal anesthesia, even with knowledge of the patient's CSF volume. Nonetheless, I expect that when technological advancements provide this measure, it will prove useful, but not by the application of theoretical models. Rather, clinical studies will inform us how to use CSF volume with other measurable patient parameters to choose a dose that is empirically known to produce a specified drug effect. After all, it has been practical experience and observation that has guided the development of spinal anesthesia, while science tries to catch up and explain its mysteries.

\section{Une tentative de com- préhension de la rachi- anesthésie}

L'administration d'une anesthésie sûre et efficace nécessite non seulement une observation vigilante, mais aussi une technologie qui nous éclaire au-delà de ce que nous pouvons percevoir naturellement avec nos sens. Dès les années 1970, la pratique de l'anesthésie est entrée dans une phase comprenant petit à petit davantage de mesures concrètes et moins de suppositions. Nous prenons désormais pour acquis que l'électrocardiographie nous donnera le rythme cardiaque, que la stimulation nerveuse révélera l'intensité du bloc neuromusculaire, que l'oxymétrie dévoilera l'oxygénation et que la capnométrie permettra de surveiller l'élimination du dioxyde de carbone. De même, la recherche d'un moniteur de la profondeur de l'anesthésie basé sur des indices électrophysiologiques est, elle aussi, poussée par notre malaise commun face au manque de mesures directes de certaines fonctions physiologiques cruciales pendant l'anesthésie.

Un mécontentement similaire quant au manque de données claires retient certains médecins et les poussent à ne pas pratiquer d'anesthésie régionale ; en effet, nous opérons à tâtons par rapport à l'emplacement des nerfs, à la diffusion de l'anesthésique, et à la grande variation dans les résultats. Tout cela explique le soupir de soulagement collectif provoqué par l'arrivée récente de l'imagerie par ultrason pour les blocs nerveux périphériques.
Malheureusement, nous demeurons dans le flou concernant certains aspects critiques des blocs neuraxiaux. Le fait qu'une dose d'anesthésique local donnée ait une diffusion très variable selon les patients est source de frustration. Nous reconnaissons que certaines catégories de patients auront en moyenne un bloc plus étendu, par exemple les femmes enceintes, les patients âgés ou obèses. Néanmoins, nous ne possédons pas de moyens pour anticiper le comportement d'un patient individuel, et c'est pourquoi nous sommes dans l'impossibilité d'appliquer des mesures qui contrôleraient l'étendue du bloc, comme choisir une dose, une gravité spécifique ou une position du patient particulières.

Un rayon de lumière a éclairé ce mystère lorsque Rosenberg et d'autres ont suivi un pressentiment clinique et confirmé qu'un patient recevant des anesthésiques rachidiens à répétition reproduit quasiment la même diffusion d'anesthésie sensitive chaque fois. ${ }^{1,2}$ Etant donné que l'influence de l'âge et l'indice de masse corporelle n'expliquent pas cette découverte, une autre caractéristique du patient doit influencer de manière critique la diffusion de l'anesthésique rachidien. Un paramètre physique évidemment pertinent est le volume de liquide céphalo-rachidien (LCR). Tout comme les doses d'agents anesthésiques locaux sont ajustées par rapport à la taille du patient afin d'obtenir les concentrations désirées dans la circulation et aux sites effecteurs, il serait adéquat d'ajuster la dose d'anesthésique local intrathécal pour convenir au volume qui le dilue initialement. La mesure directe du volume du LCR vertébral n'est devenue possible qu'avec l'invention de l'imagerie axiale, et les découvertes effectuées à l'aide de l'imagerie par résonance magnétique (IRM) confirment en effet qu'il y a notamment une distribution étendue de volumes de LCR lombaire, par exemple une gamme de $21 \mathrm{~mL}$ à $74 \mathrm{~mL}$ chez 25 patients typiques. ${ }^{3}$ Il est facile de croire que des différences si extraordinaires doivent réguler la dilution anesthésique et ainsi l'étendue du bloc, ${ }^{4}$ et que le volume du LCR d'un patient en particulier serait une mesure précieuse qu'il faudrait connaître avant la rachianesthésie. Bien que les techniques d'IRM récentes soient efficaces, ${ }^{5}$ il ne s'agit pas encore d'une modalité utile à la pratique clinique.

Ce numéro du Journal rapporte la tentative louable d'Arzola et coll. ${ }^{6}$ d'utiliser la technologie d'ultrason aisément disponible pour déterminer un index de volume du LCR. Il est décevant que le diamètre dural qu'ils ont mesuré par ultrason ne corresponde pas à l'étendue du bloc, mais certaines considérations ont rendu leur étude particulièrement ardue. Etant donné que l'indication chirurgicale était l'accouchement par 
césarienne, les auteurs ont recherché un bloc haut, et il n'est pas autrement surprenant que la hauteur maximale du bloc se soit concentrée autour de T2 et $\mathrm{T} 3$, en raison de certains traits non uniformes de l'espace sous-arachnoïdien. La quantité de LCR aux niveaux thoraciques est réduite en comparaison des niveaux lombaires et cervicaux, ${ }^{3}$ et les racines nerveuses thoraciques sont très fines comparées à celles des segments situés au-dessus et en-dessous. ${ }^{7}$ Il y a ainsi moins de dilution anesthésique par unité segmentale de distance du site d'injection, et les racines sont aisément bloquées à cause de leur petite taille; ces deux facteurs prédisent un bloc efficace de ces segments. Toutefois, plus haut, le volume de LCR accru a pour conséquence une plus grande dilution et les racines plus larges sont plus difficiles à bloquer; ceci est donc une barrière commune à la diffusion maximale d'un bloc. Dans une telle étude avec des blocs quasi-maximaux, une influence du volume diluant variable de LCR pourrait ne pas jouer un rôle prépondérant. Une deuxième limite à ces résultats est liée au niveau actuel de résolution de l'image disponible par ultrason. Sans pouvoir distinguer la dure-mère du ligament jaune, ni la quantité appréciable de graisse péridurale entre eux ${ }^{8}$ d'importantes différences dans le diamètre ont peut-être été occultées. Enfin, le sac dural n'est pas un simple cercle en coupe,${ }^{8}$ et les volumes peuvent différer alors que le diamètre dural antérieur/postérieur demeure constant.

Bien que je me réjouisse beaucoup de l'évolution continue des méthodes d'imagerie qui fourniront au bout du compte une mesure du volume du LCR, il est important de penser à ce que nous ferons une fois cette information en nos mains. Premièrement, sauronsnous quelle concentration d'anesthésique local cibler ? Certains chiffres pertinents ont déjà été avancés. Dans les corps cellulaires des neurones sensitifs qui sont extraits du ganglion de la racine postérieure par dissociation enzymatique, les canaux sodiques voltage dépendants de la membrane plasmique sont bloqués par la lidocaïne avec des concentrations $\mathrm{EC}_{50}$ de 100 $\mu \mathrm{M}$ (égales à $\left.23 \mu \mathrm{g} \cdot \mathrm{mL}^{-1}\right){ }^{9}$ Toutefois, ceci n'est pas complètement pertinent, étant donné que ces neurones isolés ne possèdent pas la couche d'encerclement de cellules satellites qui sont présentes in vivo dans le ganglion. De plus, les axones des neurones sensitifs expriment une surabondance de canaux, de sorte que des potentiels d'action puissent avoir lieu même si la plupart des canaux $\mathrm{Na}^{+}$sont bloqués. Pour ces raisons, la concentration de lidocaïne nécessaire à l'interruption de la conduction des potentiels d'action dans des racines postérieures excisées montre un $\mathrm{EC}_{50}$ d'environ $230 \mu \mathrm{M}\left(54 \mu \mathrm{g} \cdot \mathrm{mL}^{-1}\right)$, et un bloc complet néces- site plus de $500 \mu \mathrm{M}\left(115 \mu \mathrm{g} \cdot \mathrm{mL}^{-1}\right) .{ }^{10}$ Etant donné que ces études utilisent des tissus de rats plutôt que d'humains, et étant donné que des chambres in vitro sont très différentes de l'environnement intrathécal, la recherche sur les cellules devra en fin de compte être validée par des recherches sur les humains. Dans ce domaine, les données expérimentales sont malheureusement rares et remarquablement vieilles, bien qu'elles apparaissent toutefois très précises. Des travaux historiques commencés dans les années $1930^{11-13}$ ont utilisé l'échantillonnage périodique de LCR par cathéter ou aiguille malléable pour cartographier la distribution et l'action de l'anesthésique, examinant à l'origine la procaïne, l'agent anesthésique courant à l'époque. Des recherches ultérieures sur les anesthésiques rachidiens à base de lidocaïne ${ }^{14}$ ont trouvé des concentrations au retour de la sensation $\left(1,07 \mathrm{mM}, 250 \mu \mathrm{g} \cdot \mathrm{mL}^{-1}\right)$ et du mouvement $\left(1,40 \mathrm{mM}, 327 \mu \mathrm{g} \cdot \mathrm{mL}^{-1}\right)$ qui coïncident très bien avec les concentrations utilisées pour un bloc complet dans les expérimentations animales modernes. ${ }^{10,15}$ (Dans leur ensemble, les études menées par les premiers chercheurs en anesthésie régionale inspirent beaucoup d'admiration quant à l'exactitude de leurs découvertes).

Même si nous avions ces données, un autre défi se présente à nous. Serions-nous capables de créer la concentration anesthésique neuronale désirée si nous connaissions le volume de LCR du patient? Ici, le problème réside en ce que la pharmacocinétique du LCR est très complexe $e^{16}$ en raison de l'action simultanée de nombreux processus. Bien que la distribution du médicament soit facilitée par les oscillations pulsatiles du LCR ${ }^{17}$ (reconnu comme un facteur important par Koster et coll. ${ }^{13}$ en 1936), le LCR n'est pas bien mélangé. Au contraire, il y a une chute abrupte de la concentration à mesure que la distance grandit depuis le point d'injection. ${ }^{12,18,19}$ Cette caractéristique est en opposition à celle de la pharmacocinétique de l'administration systémique de médicament, par laquelle les concentrations deviennent rapidement uniformes dans le volume sanguin. L'influence de la baricité ajoute encore à la distribution non uniforme du médicament dans le LCR. L'élimination des médicaments injectés dans le LCR est également complexe sur le plan du temps, étant donné que le transport médicamenteux du LCR vers le tissu nerveux entre en conflit avec le captage vasculaire et le départ du médicament vers l'espace péridural. Enfin, un état stable du patient n'est pas obtenu dans ce système dynamique, et certaines indications laissent à penser que la concentration maximale qui donne l'impulsion initiale à l'agent anesthésique local vers les racines nerveuses pourrait avoir davantage d'influence sur l'étendue et 
la durée du bloc que les concentrations instantanées présentes plus tard. ${ }^{14}$ Bien que certaines simulations de la performance pharmacocinétique du LCR utilisant des modèles multi-compartimentaux ${ }^{20,21}$ aient déjà été effectuées, celles-ci reposent en fin de compte sur la compatibilité empirique post hoc d'un nombre incertain d'éléments exponentiels contenus dans les données.

Une découverte cohérente que nous avons faite après l'injection de médicaments dans l'espace sousarachnoïdien consiste en l'extrême variabilité interindividuelle dans les concentrations mesurées. Dans de petits groupes de patients, des différences de concentration de trois à dix fois plus importantes sont observées après injection d'une même dose ${ }^{14,18,19,21,22}$ et ce, même durant les premières dix à 30 min pendant lesquelles le mélange initial est atteint. ${ }^{23}$ Je soupçonne que cela est en partie dû au volume très variable de LCR, mais également à l'inhomogénéité de l'espace. Plutôt que la chambre ouverte que nous connaissons dans les descriptions communes et les modèles de verre, l'espace sous-arachnoïdien postérieur est divisé par une quantité de membranes, ${ }^{24-27}$ lesquelles apparaissent le mieux dans les spécimens d'autopsie non embaumés. Un anesthésique injecté dans ce système complexe et multi-chambres pourrait pénétrer le bassin général du LCR lombaire ou, dans d'autres cas, demeurer relativement isolé, ce qui pourrait résulter en des degrés de dilution différents.

Ces observations prédisent qu'il sera difficile de calculer une dose d'anesthésique local produisant exactement la distribution et la durée de rachianesthésie désirées, même en connaissant le volume de LCR du patient. Néanmoins, je m'attends à ce que cela soit utile lorsque les progrès technologiques fourniront ces mesures, mais pas par l'application des modèles théoriques. En revanche, les études cliniques nous éclaireront sur la manière de se servir du volume de LCR conjointement à d'autres paramètres mesurables relatifs au patient, afin de choisir une dose dont la connaissance empirique assure qu'elle produira un effet médicamenteux spécifique. Après tout, c'est l'expérience pratique et l'observation qui ont guidé le développement de la rachianesthésie, alors que la science tente de rattraper le retard et d'expliquer ses mystères.

\section{References}

1 Taivainen TR, Tuominen MK, Kuulasmaa KA, Rosenberg $P H$. A prospective study on reproducibility of the spread of spinal anesthesia using plain $0.5 \%$ bupivacaine. Reg Anesth 1990; 15: 12-4.

2 Tuominen M, Kuulasmaa K, Taivainen T, Rosenberg
PH. Individual predictability of repeated spinal anaesthesia with isobaric bupivacaine. Acta Anaesthesiol Scand 1989; 33: 13-4.

3 Hogan QH, Prost R, Kulier A, Taylor ML, Liu S, Mark $L$. Magnetic resonance imaging of cerebrospinal fluid volume and the influence of body habitus and abdominal pressure. Anesthesiology 1996; 84: 1341-9.

4 Carpenter RL, Hogan QH, Liu SS, Crane B, Moore J. Lumbosacral cerebrospinal fluid volume is the primary determinant of sensory block extent and duration during spinal anesthesia. Anesthesiology 1998; 89: 24-9.

5 Sullivan JT, Grouper S, Walker MT, Parrish TB, McCarthy RJ, Wong CA. Lumbosacral cerebrospinal fluid volume in humans using three-dimensional magnetic resonance imaging. Anesth Analg 2006; 103: 1306-10.

6 Arzola C, Balki M, Carvalho JC. The antero-posterior diameter of the lumbar dural sac does not predict sensory levels of spinal anesthesia for cesarean delivery. Can J Anesth 2007; 54: 620-5.

7 Hogan Q. Size of human lower thoracic and lumbosacral nerve roots. Anesthesiology 1996; 85: 37-42.

8 Hogan QH. Lumbar epidural anatomy. A new look by cryomicrotome section. Anesthesiology 1991; 75: 767-75.

9 Gold MS, Reichling DB, Hampl KF, Drasner K, Levine $J D$. Lidocaine toxicity in primary afferent neurons from the rat. J Pharmacol Exp Ther 1998; 285: 413-21.

10 Jaffe RA, Rowe MA. Differential nerve block. Direct measurements on individual myelinated and unmyelinated dorsal root axons. Anesthesiology 1996; 84: 1455-64.

11 Frumin MJ, Schwartz H, Burns JJ, Brodie BB, Papper $E M$. Sites of sensory blockade during segmental spinal and segmental peridural anesthesia in man. Anesthesiology 1953; 14: 576-83.

12 Helrich M, Papper EM, Brodie BB, Fink M, Rovenstine $E A$. The fate of intrathecal procaine and the spinal fluid level required for surgical anesthesia. J Pharmacol Exp Ther 1950; 100: 78-82.

13 Koster H, Shapiro A, Leikensohn A. Spinal anesthesia: procain concentration changes at site of injection in subarachnoid anesthesia. Am J Surg 1936; 33: 245-8.

14 Morch ET, Rosenberg MK, Truant AT. Lidocaine for spinal anesthesia. Acta Anaesth Scandinav 1957; 1: 105-15.

15 Popitz-Bergez FA, Leeson S, Strichartz GR, Thalhammer $J G$. Relation between functional deficit and intraneural local anesthetic during peripheral nerve block. A study in the rat sciatic nerve. Anesthesiology 1995; 83: 583-92.

16 Wood JH. Physiology, Pharmacology and Dynamics of Cerebrospinal Fluid, Neurobiology of Cerebrospinal 
Fluid. NY: Plenum Press; 1980: 1-16.

17 Enzmann DR, Pelc NJ. Normal flow patterns of intracranial and spinal cerebrospinal fluid defined with phase-contrast cine MR imaging. Radiology 1991; 178: 467-74.

18 Frumin MJ, Schwartz H, Burns J, Brodie BB, Papper $E M$. Dorsal root ganglion blockade during threshold segmental spinal anesthesia in man. J Pharmacol Exp Ther 1954; 112: 387-92.

19 Koster H, Shapiro A, Leikensohn A. Concentration of procaine in the cerebrispinal fluid of the human being after subarachnoid injection. Am J Surg 1938; 37: 603-8.

20 Shafer SL, Eisenach JC, Hood DD, Tong C. Cerebrospinal fluid pharmacokinetics and pharmacodynamics of intrathecal neostigmine methylsulfate in humans. Anesthesiology 1998; 89: 1074-88.

21 Sjostrom S, Tamsen A, Persson MP, Hartvig P. Pharmacokinetics of intrathecal morphine and meperidine in humans. Anesthesiology 1987; 67: 889-95.

22 Hansdottir V, Hedner T, Woestenborghs R, Nordberg $G$. The CSF and plasma pharmacokinetics of sufentanil after intrathecal administration. Anesthesiology 1991; 74: 264-9.

23 Van Zundert AA, Grouls RJ, Korsten HH, Lambert $D H$. Spinal anesthesia. Volume or concentration--what matters? Reg Anesth 1996; 21: 112-8.

24 Di Chiro G, Timins EL. Supine myelography and the septum posticum. Radiolgy 1974; 111: 319-27.

25 Jirout J. Pneumomyelography, 2nd ed. Springfield, IL: Charles C. Thomas Publisher; 1969: 27-41.

26 Key EA, Retzius MG. Studien in der Anatomie des Nervensystems und des Bindegewebs. Stockholm, Samson and Wallin, 1875.

27 Nauta HJ, Dolan E, Yasargil MG. Microsurgical anatomy of spinal subarachnoid space. Surg Neurol 1983; 19: 431-7. 